\title{
Representations of the Schrödinger algebra and Appell systems
}

\author{
Philip Feinsilver*, Jerzy Kocik ${ }^{\dagger}$ and René Schott ${ }^{\ddagger}$
}

\begin{abstract}
We investigate the structure of the Schrödinger algebra and its representations in a Fock space realized in terms of canonical Appell systems. Generalized coherent states are used in the construction of a Hilbert space of functions on which certain commuting elements act as self-adjoint operators. This yields a probabilistic interpretation of these operators as random variables. An interesting feature is how the structure of the Lie algebra is reflected in the probability density function. A Leibniz function and orthogonal basis for the Hilbert space is found. Then Appell systems connected with certain evolution equations, analogs of the classical heat equation, on this algebra are computed.
\end{abstract}

Keywords: Lie algebras, Schrödinger algebra, Heisenberg-Weyl algebra, Leibniz function, quantum probability, Appell systems

AMS classification: 17B81, 60BXX, 81R05

\footnotetext{
*Department of Mathematics Southern Illinois University Carbondale, IL. 62901, U.S.A. ${ }^{\dagger}$ Department of Mathematics Southern Illinois University Carbondale, IL. 62901, U.S.A. ¥IECN and LORIA Université Henri Poincaré-Nancy 1, BP 239, 54506 Vandoeuvre-lès-Nancy, France.
} 


\section{Contents}

1 Introduction 3

2 Appell systems: some interpretations 3

3 Schrödinger algebra $\quad 6$

3.1 Structural decomposition for Fock calculus . . . . . . . . . 7

3.2 A matrix representation and group calculations . . . . . . . 8

3.3 Standard form of the Schrödinger algebra . . . . . . . . . 10

4 Canonical Appell systems for the Schrödinger algebra 13

4.1 Adjoint operators and Appell systems . . . . . . . . . . . . . 14

4.2 Probability distributions . . . . . . . . . . . . . 15

$\begin{array}{lll}5 & \text { Leibniz function and orthogonal basis } & 17\end{array}$

6 Evolution equations and Appell systems 21 


\section{Introduction}

The Schrödinger Lie algebra plays an important role in mathematical physics and its applications (see, e.g., $[1,2,3,8,10]$ ). Using the technique of singular vectors, a classification of the irreducible lowest weight representations of this algebra is given in [5]. A main feature of the present paper is a classification of the representations of the Schrödinger algebra in an alternative way based on the semidirect product structure of the algebra.

We begin in $\S 2$ with some interpretations of the notion of 'Appell systems'. Section 3 contains basic details of our approach to representations of the Schrödinger algebra. In particular, we show how it is built and determine a standard form. Some group calculations are done using a matrix realization of the algebra. In $\S 4$ we construct canonical Appell systems and find a family of probability distributions associated to the Schrödinger algebra that reflects its Lie algebraic structure. In particular, we see that the results of [5] on polynomial representations based on lowest weight modules fit into our picture. The details of the associated Hilbert space comprise $\S 5$. This starts with computing the Leibniz function. We show how to recover the Lie algebra from the Leibniz function and obtain an orthogonal basis for the Hilbert space. In the final section, we show how to construct Appell systems which

provide solutions to generalized heat equations on the Schrödinger algebra, corresponding to classical two-dimensional real Lévy processes.

\section{Appell systems: some interpretations}

There are three interpretations of the notion of 'Appell systems':

1. Appell systems in the classical sense. These are considered below from a more general viewpoint.

2. Canonical Appell systems associated to a Lie algebra. One uses the Lie algebra to construct a Hilbert space with the Appell system as basis. See $\S 4$.

3. General Appell systems on Lie groups. Here one uses the Lie algebra and group structure as a 'black box' into which a classical stochastic 
process goes in and produces a 'Lie response' - typically a process consisting of iterated stochastic integrals of the input process $([6,7])$. In this paper, for simplicity, we restrict to the abelian case. Appell systems provide solutions to evolution equations related to the input stochastic process. See $\S 6$.

Now we shall expand on the first point of view.

Considering the differentiation operator $D$, we may think of the space of polynomials of degree not exceeding $n$ as the space of solutions, $\mathcal{Z}_{n}$, to the equation $D^{n+1} \psi=0$. In this context an Appell system is defined to be a sequence of nonzero polynomials $\left\{\psi_{0}, \psi_{1}, \ldots, \psi_{n}, \ldots\right\}$ satisfying:

1. $\psi_{n} \in \mathcal{Z}_{n}, \forall n \geq 0$

2. $D \psi_{n}=\psi_{n-1}$, for $n \geq 1$

(Note that this differs slightly from the usual definition, cf. [6], which has $D \psi_{n}=n \psi_{n-1}$.) By analogy, for any operator $V$, called the canonical lowering operator, we define a $V$-Appell system as follows. Set

$$
\mathcal{Z}_{n}=\left\{\psi: V^{n+1} \psi=0\right\}
$$

for $n \geq 0$. Then the $V$-Appell space decomposition is the system of embeddings $\mathcal{Z}_{0} \subset \mathcal{Z}_{1} \subset \mathcal{Z}_{2} \subset \ldots$, and a $V$-Appell system is a sequence of nonzero functions $\left\{\psi_{0}, \psi_{1}, \ldots, \psi_{n}, \ldots\right\}$ satisfying:

1. $\psi_{n} \in \mathcal{Z}_{n}, \forall n \geq 0$

2. $V \psi_{n}=\psi_{n-1}$, for $n \geq 1$

Typically, one starts with a 'standard Appell system', such as $\psi_{n}=x^{n} / n$ !, for $V=D$. Then Appell systems are generated from the standard one via time-evolution. To accomplish this for $V$-Appell systems, the symmetry algebra of $V$ comes into play.

If $V$ is an operator acting on a space of smooth functions, we define its unrestricted symmetry algebra to be the Lie algebra $\mathfrak{g}(V)$ of vector fields, $X$, such that there exists an operator $\Lambda(X)$ in the center of $\mathfrak{g}(V)$ with

$$
[X, V]=\Lambda(X) V
$$


If in every case we require $\Lambda$ to be multiplication by a scalar function, we shall talk of the restricted symmetry algebra, as in [5]. If we consider only those $X$ for which $\Lambda(X)=0$, we have the strict symmetry algebra $\mathfrak{g}_{0}(V) \subset \mathfrak{g}(V)$. Clearly, $V \in \mathfrak{g}_{0}(V)$. Also, it is clear that $\mathfrak{g}_{0}(V)$ contains the center of $\mathfrak{g}(V)$.

Proposition 2.1 The strict symmetry algebra contains the derived algebra of unrestricted symmetries: $\mathfrak{g}^{\prime}(V) \subset \mathfrak{g}_{0}(V)$. That is, $Y \in \mathfrak{g}^{\prime}(V)$ implies $[Y, V]=0$.

Proof: Let $\left[X_{1}, V\right]=\Lambda\left(X_{1}\right) V$ and $\left[X_{2}, V\right]=\Lambda\left(X_{2}\right) V$. Then, by the Jacobi identity,

$$
\begin{aligned}
{\left[\left[X_{1}, X_{2}\right], V\right] } & =\left[X_{1}, \Lambda\left(X_{2}\right) V\right]+\left[\Lambda\left(X_{1}\right) V, X_{2}\right] \\
& =\Lambda\left(X_{2}\right)\left[X_{1}, V\right]+\Lambda\left(X_{1}\right)\left[V, X_{2}\right] \\
& =\Lambda\left(X_{2}\right) \Lambda\left(X_{1}\right) V-\Lambda\left(X_{1}\right) \Lambda\left(X_{2}\right) V=0
\end{aligned}
$$

using the property that the $\Lambda$ operators are central.

The relevance for $V$-Appell systems is this.

Proposition 2.2 The unrestricted symmetry algebra $\mathfrak{g}(V)$ of an operator $V$ preserves the Appell space decomposition $\mathcal{Z}_{0} \subset \mathcal{Z}_{1} \subset \mathcal{Z}_{2} \subset \ldots$, that is, $X \mathcal{Z}_{n} \subset \mathcal{Z}_{n}$ for every $X \in \mathfrak{g}(V)$.

Proof: Write $[X, V]=\Lambda(X) V$ in the form $V X=(X-\Lambda(X)) V$. Fix $n \geq 0$ and let $\psi \in \mathcal{Z}_{n}$. Since $\Lambda(X)$ commutes with $V$, we have $V^{n+1} X \psi=$ $(X-(n+1) \Lambda(X)) V^{n+1} \psi=0$.

New Appell systems are generated from a given one by the adjoint action of a group element generated by a 'Hamiltonian' — a function of elements of the symmetry algebra. The structure of the spaces $\mathcal{Z}_{n}$ is preserved, while the Appell systems provide 'polynomial solutions' to the evolution equation corresponding to the Hamiltonian. Indeed, if $H$ is a function of operators in $\mathfrak{g}(V)$, with $H \psi_{0}=0$, then $h_{n}=\exp (t H) \psi_{n}$ will be an Appell system. For each $n$, the function $h_{n}$ satisfies $u_{t}=H u$, with $u(0)=\psi_{n}$. In the simplest situation where $H$ is a function of $D$ and the initial Appell sequence is $\psi_{n}=x^{n} / n$ !, different choices of $H$ yield many of the classically important 
sequences of polynomials (with perhaps minor variations).

In the paper [5], a hierarchy of solutions to $\mathbf{S}^{(p / 2)} \psi=0$ is developed for the Schrödinger operator $\mathbf{S}$. The representations discussed there can be viewed as S-Appell systems in the above sense. These correspond to finite-dimensional representations of $\operatorname{sl}(2)$ in the standard form of the Schrödinger algebra given below.

\section{Schrödinger algebra}

Referring to [5] for details, recall that the ( $n=1$, centrally-extended) Schrödinger algebra $\mathcal{S}_{1}$ is spanned by the following elements (commented by their physical origins):

$\begin{array}{cl}M & \text { mass } \\ K & \text { special conformal transformation } \\ G & \text { Galilei boost } \\ D & \text { dilation (not differentiation!) } \\ P_{x} & \text { spatial translation } \\ P_{t} & \text { time translation }\end{array}$

which satisfy the following commutation relations, given here in the form of a matrix with rows and columns labelled by the corresponding operators

\begin{tabular}{|c|c|c|c|c|c|}
\hline$M$ & $K$ & $G$ & $D$ & $P_{x}$ & $P_{t}$ \\
\hline$M$ & 0 & 0 & 0 & 0 & 0 \\
\hline$K$ & 0 & 0 & $-2 K$ & $-G$ & $-D$ \\
\hline$G$ & 0 & 0 & $-G$ & $-M$ & $-P_{x}$ \\
\hline$D$ & $2 K$ & $G$ & 0 & $-P_{x}$ & $-2 P_{t}$ \\
\hline$P_{x}$ & $G$ & $M$ & $P_{x}$ & 0 & 0 \\
\hline$P_{t} \backslash 0$ & $D$ & $P_{x}$ & $2 P_{t}$ & 0 & 0 \\
\hline
\end{tabular}

Note that elements $\left\{M, G, P_{x}\right\}$ span a Heisenberg-Weyl subalgebra, while $\left\{K, D, P_{t}\right\}$ span an sl(2) subalgebra. This fact, that the Schrödinger algebra 
is a semidirect product

$$
\mathcal{S}_{1} \cong \mathcal{H} \oplus_{s} \operatorname{sl}(2)
$$

is the basis for analyzing the representations of the Schrödinger algebra. We continue with the $n=1$ case and indicate how the general case $n>1$ goes at the end of the discussion of the standard form, since the rotation generators, $J_{i j}$, do not appear in the case $n=1$.

\subsection{Structural decomposition for Fock calculus}

In general, in order to construct representations, we first seek a generalized Cartan decomposition of the Schrödinger algebra into a triple $\mathfrak{g}=\mathcal{P} \oplus \mathcal{K} \oplus \mathcal{L}$ where $\mathcal{P}$ and $\mathcal{L}$ are abelian subalgebras, and $\mathcal{K}$ is a subalgebra normalizing both $\mathcal{P}$ and $\mathcal{L}$. The main idea is that elements of $\mathcal{P}$ and $\mathcal{L}$ act as raising and lowering operators, respectively. The possibility of finding a scalar product in which each element of $\mathcal{P}$ has a corresponding adjoint in $\mathcal{L}$ is important, since we wish to construct a family of selfadjoint operators that provide a family of commuting quantum observables or classical random variables in the probabilistic interpretation. In many cases, this family arises by conjugating elements of $\mathcal{P}$ by a group element with a generator from $\mathcal{L}$. This technique may be viewed as an extension of the Cayley transform for symmetric spaces. Notice that for this to work, the subalgebras $\mathcal{P}$ and $\mathcal{L}$ must be in one-to-one correspondence - the Cartan involution in the theory of symmetric spaces.

The Schrödinger algebra $\mathfrak{g}=\mathcal{S}_{1}$ admits the following generalized Cartan decomposition:

$$
\{m, K, G\} \oplus\left\{D, P_{x}\right\} \oplus\left\{P_{t}\right\}
$$

Note however that $\mathcal{P}$ and $\mathcal{L}$ cannot be put into 1-1 correspondence and therefore this is of no direct use for us.

We will use instead the following decomposition ( cf. [9, p. 31]):

$$
\underbrace{\{K, G\}}_{\mathcal{P}} \oplus \underbrace{\{M, D\}}_{\mathcal{K}} \oplus \underbrace{\left\{P_{t}, P_{x}\right\}}_{\mathcal{L}}
$$

$M$ acts here as a scalar $m$. We take $R_{1}=K$ and $R_{2}=G$ as raising operators. Even though $P_{x}$ is not in "Cartan's $\mathcal{L}$ ", as in equation $(1)$, we use it as the 
lowering operator dual to $G$, so take $L_{1}=P_{t}$ and $L_{2}=P_{x}$.

Even though the decomposition (2) is not technically a Cartan decomposition, it will lead to interesting results for representations of the Schrödinger algebra.

\subsection{A matrix representation and group calculations}

A 4-dimensional representation (see [4]) of the Schrödinger algebra $(n=1)$ is given by embedding into $\mathrm{su}(4)$. Let $X$ denote a typical element of the Lie algebra. Set,

$$
X=a_{1} m+a_{2} K+a_{3} G+a_{4} D+a_{5} P_{x}+a_{6} P_{t}=\left(\begin{array}{cccc}
0 & a_{5} & a_{3} & 2 a_{1} \\
0 & a_{4} & a_{2} & a_{3} \\
0 & -a_{6} & -a_{4} & -a_{5} \\
0 & 0 & 0 & 0
\end{array}\right)
$$

We will denote a typical group element according to the basis we have chosen by

$$
\begin{aligned}
& g\left(A_{1}, A_{2}, A_{3}, A_{4}, A_{5}, A_{6}\right)= \\
& \quad \exp \left(A_{1} m\right) \exp \left(A_{2} K\right) \exp \left(A_{3} G\right) \exp \left(A_{4} D\right) \exp \left(A_{5} P_{x}\right) \exp \left(A_{6} P_{t}\right)
\end{aligned}
$$

The variables $\left\{A_{i}\right\}$ are coordinates of the second kind. The group element corresponding to $(3)$ is

$g\left(A_{1}, A_{2}, A_{3}, A_{4}, A_{5}, A_{6}\right)=e^{-A_{4}} \cdot\left(\begin{array}{cccc}e^{A_{4}} & A_{5} e^{A_{4}}-A_{3} A_{6} & A_{3} & 2 A_{1} e^{A_{4}}-A_{3} A_{5} \\ 0 & e^{2 A_{4}}-A_{2} A_{6} & A_{2} & -A_{3} e^{A_{4}}-A_{2} A_{5} \\ 0 & -A_{6} & 1 & -A_{5} \\ 0 & 0 & 0 & e^{A_{4}}\end{array}\right)$

From this we have

Proposition 3.1 Given in matrix form a group element $g$, we can recover the second-kind coordinates $\left(A_{1}, \ldots, A_{6}\right)$ according to

$$
\begin{array}{cc}
A_{1}=-\frac{1}{2} \frac{\left|\begin{array}{ll}
g_{13} & g_{14} \\
g_{33} & g_{34}
\end{array}\right|}{g_{33}}, & A_{2}=\frac{g_{23}}{g_{33}}, \quad A_{3}=\frac{g_{13}}{g_{33}}, \\
A_{4}=-\log \left(g_{33}\right), & A_{5}=-\frac{g_{34}}{g_{33}}, \quad A_{6}=-\frac{g_{32}}{g_{33}}
\end{array}
$$


Referring to decomposition (2), we specialize variables, writing $V_{1}, V_{2}, B_{1}, B_{2}$ for $A_{2}, A_{3}, A_{6}, A_{5}$ respectively. Basic for our analysis is the partial group law:

$$
e^{B_{1} P_{t}+B_{2} P_{x}} e^{V_{1} K+V_{2} G}=?
$$

We will get the required results using the matrix representation noted above. The general elements of $\mathcal{P}$ and $\mathcal{L}$ are:

$$
\begin{aligned}
B_{1} P_{t}+B_{2} P_{x} & =\left(\begin{array}{cccc}
0 & B_{2} & 0 & 0 \\
0 & 0 & 0 & 0 \\
0 & -B_{1} & 0 & -B_{2} \\
0 & 0 & 0 & 0
\end{array}\right) \\
V_{1} K+V_{2} G & =\left(\begin{array}{cccc}
0 & 0 & V_{2} & 0 \\
0 & 0 & V_{1} & V_{2} \\
0 & 0 & 0 & 0 \\
0 & 0 & 0 & 0
\end{array}\right)
\end{aligned}
$$

As the square of each of these matrices is zero, the exponential of each reduces to simply adding the identity. We find the matrix product

$$
e^{B_{1} P_{t}+B_{2} P_{x}} e^{V_{1} K+V_{2} G}=\left(\begin{array}{cccc}
1 & B_{2} & V_{2}+B_{2} V_{1} & B_{2} V_{2} \\
0 & 1 & V_{1} & V_{2} \\
0 & -B_{1} & 1-B_{1} V_{1} & -B_{1} V_{2}-B_{2} \\
0 & 0 & 0 & 1
\end{array}\right)
$$

Applying Proposition 3.1 to the matrix found above yields

Proposition 3.2 In coordinates of the second kind, we have the Leibniz formula,

$$
\begin{aligned}
& g\left(0,0,0,0, B_{2}, B_{1}\right) g\left(0, V_{1}, V_{2}, 0,0,0\right)= \\
& g\left(\frac{1}{2} \frac{B_{1} V_{2}^{2}+2 B_{2} V_{2}+B_{2}^{2} V_{1}}{1-B_{1} V_{1}}, \frac{V_{1}}{1-B_{1} V_{1}}, \frac{V_{2}+B_{2} V_{1}}{1-B_{1} V_{1}},\right. \\
& \left.-\log \left(1-B_{1} V_{1}\right), \frac{B_{1}+V_{2}}{1-B_{1} V_{1}}, \frac{B_{1}}{1-B_{1} V_{1}}\right)
\end{aligned}
$$

In general, a Leibniz formula is the group law for commuting the $L$ operators past the $R$ 's, in analogy to the classical formula of Leibniz for derivatives. 


\subsection{Standard form of the Schrödinger algebra}

Now we show the internal structure of the Schrödinger algebra $(n=1)$.

Remark 3.3 Note that we work in enveloping algebras throughout, so our calculations are based on relations in an associative algebra. In particular, we often use

$$
[A, B C]=[A, B] C+B[A, C] \quad \text { and } \quad\left[A, B^{2}\right]=[A, B] B+B[A, B]
$$

Definition 3.4 Denote the basis for a standard Heisenberg-Weyl (HW) algebra, $\mathcal{H}=\operatorname{span}\{P, X, H\}$, satisfying

$$
[P, X]=H, \quad[P, H]=[X, H]=0
$$

A representation of $\mathrm{HW}$-algebra such that $H$ acts as the scalar $m$ times the identity operator will be denoted as $m$-HW algebra.

Definition 3.5 Denote the basis for a standard $\mathrm{sl}(2)$ algebra, $\mathcal{K}$, by $\{L, R, \rho\}$, satisfying

$$
[L, R]=\rho, \quad[\rho, R]=2 R, \quad[L, \rho]=2 L
$$

We write $\mathcal{K}:=\{L, R, \rho\}$.

The following Lemma is well-known. It follows readily from the equations in remark 3.3 (also see calculations below).

Lemma 3.6 Given an m-HW algebra, setting

$$
L=\frac{1}{2 m} P^{2}, \quad \rho=\frac{1}{m} X P+\frac{1}{2}, \quad R=\frac{1}{2 m} X^{2}
$$

yields a standard $\mathrm{sl}(2)$ algebra.

Now for our first main observation, which follows immediately from the commutation rules for the Schrödinger algebra.

Theorem 3.7 (HW form of the Schrödinger algebra) Given an $m$-HW algebra, setting

$$
m=H, K=\frac{1}{2 m} X^{2}, G=X, D=\frac{1}{m} X P+\frac{1}{2}, P_{x}=P, P_{t}=\frac{1}{2 m} P^{2}
$$

yields a representation of $\mathcal{S}$. 
And the main theorem, which gives the standard form.

Theorem 3.8 (Standard form of the Schrödinger algebra) Any representation of the Schrödinger algebra $\mathcal{S}=\operatorname{span}\left\{m, K, G, D, P_{x}, P_{t}\right\}$ contains a standard $\operatorname{sl}(2)$ algebra $\mathcal{K}_{0}=\operatorname{span}\left\{L_{0}, R_{0}, \rho_{0}\right\}$ such that, with the $m-\mathrm{HW}$ algebra $\mathcal{H}=\operatorname{span}\left\{P_{x}, G, m\right\}$ from the given representation of $\mathcal{S}$, the $\operatorname{sl}(2)$ subalgebra is of the form

$$
K=R_{0}+\frac{1}{2 m} G^{2}, \quad D=\rho_{0}+\frac{1}{m} G P_{x}+\frac{1}{2}, \quad P_{t}=L_{0}+\frac{1}{2 m} P_{x}^{2}
$$

where $\mathcal{K}_{0}$ commutes with $\mathcal{H}$.

Conversely, given any $m$-HW representation, use it for $\mathcal{H}:=\left\{P_{x}, G, m\right\}$. Now take any $\mathrm{sl}(2)$ algebra commuting with $\mathcal{H}$, and form the direct product with the standard $\mathrm{sl}(2)$ algebra constructed from $\mathcal{H}$ by the Lemma. Then this yields a representation of $\mathcal{S}$.

Proof: The converse is clear by construction and our previous observations. What must be checked is that given a representation of $\mathcal{S}$, setting

$$
R_{0}=K-\frac{1}{2 m} G^{2}, \rho_{0}=D-\left(\frac{1}{m} G P_{x}+\frac{1}{2}\right), L_{0}=P_{t}-\frac{1}{2 m} P_{x}^{2}
$$

yields an $\operatorname{sl}(2)$ algebra that commutes with $\mathcal{H}$. From equation (4), we have

$$
\left[L_{0}, G\right]=\left[P_{t}, G\right]-\frac{1}{2 m}\left[P_{x}^{2}, G\right]=P_{x}-P_{x}=0
$$

and similar relations for $R_{0}$ and $\rho_{0}$ show that $\mathcal{K}_{0}$ commutes with $\mathcal{H}$. Now, using remark 3.3, we note these relations

$$
\begin{aligned}
{\left[P_{x}^{2}, K\right] } & =P_{x} G+G P_{x}=2 G P_{x}+m \\
{\left[G P_{x}, K\right] } & =0 \cdot P_{x}+G \cdot G=G^{2} \\
{\left[P_{t}, G P_{x}\right] } & =P_{x}^{2}
\end{aligned}
$$

Thus, using the fact that $\left[\mathcal{K}_{0}, \mathcal{H}\right]=0$, we have

$$
\begin{aligned}
{\left[L_{0}, R_{0}\right] } & =\left[L_{0}, K-\frac{1}{2 m} G^{2}\right] \\
& =\left[P_{t}-\frac{1}{2 m} P_{x}^{2}, K\right]+\left[L_{0},-\frac{1}{2 m} G^{2}\right] \\
& =D-\frac{1}{m} G P x-\frac{1}{2}=\rho_{0}
\end{aligned}
$$


while

$$
\begin{aligned}
{\left[\rho_{0}, R_{0}\right] } & =\left[\rho_{0}, K-\frac{1}{2 m} G^{2}\right]=\left[\rho_{0}, K\right] \\
& =[D, K]-\frac{1}{m}\left[G P_{x}, K\right] \\
& =2 K-\frac{1}{m} G^{2}=2 R_{0}
\end{aligned}
$$

and

$$
\begin{aligned}
{\left[L_{0}, \rho_{0}\right] } & =\left[P_{t}-\frac{1}{2 m} P_{x}^{2}, \rho_{0}\right] \\
& =\left[P_{t}, D\right]-\left[P_{t}, \frac{1}{m} G P_{x}\right] \\
& =2 P_{t}-\frac{1}{m} P_{x}^{2}=2 L_{0}
\end{aligned}
$$

which completes the proof.

Remark 3.9 The theorem, extended to include rotations, holds also for $n>$ 1 , where we use $\mathcal{K}_{0}$ spanned by

$$
L_{0}=\frac{1}{2 m} \sum_{i} P_{i}^{2}, \quad R_{0}=\frac{1}{2 m} \sum_{i} G_{i}^{2}, \quad \rho_{0}=\frac{1}{m} \sum_{i} G_{i} P_{i}+\frac{n}{2}
$$

and for the rotations,

$$
J_{0, i j}=J_{i j}-\frac{1}{m}\left(G_{i} P_{j}-G_{j} P_{i}\right)
$$

with the $J_{0}$ rotations commuting with $\mathcal{H}$.

As an application of Theorem 3.7, consider the special realization, with scalar $M=m$ and $x$ denoting multiplication by the variable $x$,

$$
G=m x, \quad P_{x}=\frac{d}{d x}, \quad P_{t}=\frac{1}{2 m} \frac{d^{2}}{d x^{2}}, \quad K=\frac{m x^{2}}{2}, \quad D=x \frac{d}{d x}+\frac{1}{2}
$$


In this realization, acting on the function identically equal to 1 , we have $P_{t} 1=P_{x} 1=0$, and $D 1=1 / 2$. Applying a group element to the function 1 , we find

$$
g\left(A_{1}, A_{2}, A_{3}, A_{4}, A_{5}, A_{6}\right) 1=\exp \left(A_{1} m+A_{2} \frac{m x^{2}}{2}+A_{3} m x+A_{4} / 2\right)
$$

Clearly, $f(x) 1$ can be identified with the function $f(x)$ itself. Now apply the Leibniz formula, Proposition 3.2, to find

Corollary 3.10 The differential realization of the Schrödinger algebra $\mathcal{S}_{1}$ has the following "partial group law"

$$
\begin{aligned}
\exp \left(\frac{B_{1}}{2 m}\right. & \left.\frac{d^{2}}{d x^{2}}+B_{2} \frac{d}{d x}\right) \exp \left(V_{1} \frac{m x^{2}}{2}+V_{2} m x\right) \\
= & \exp \left(\frac{V_{1}}{1-B_{1} V_{1}} \frac{m x^{2}}{2}+\frac{V_{1} B_{2}+V_{2}}{1-B_{1} V_{1}} m x\right) \\
& \times\left(1-B_{1} V_{1}\right)^{-1 / 2} \exp \left(\frac{m}{2} \frac{B_{1} V_{2}^{2}+2 B_{2} V_{2}+B_{2}{ }^{2} V_{1}}{1-B_{1} V_{1}}\right)
\end{aligned}
$$

\section{Canonical Appell systems for the Schrödinger algebra}

Now to construct the representation space and basis - the canonical Appell system. To start, define a vacuum state $\Omega$ such that, for constants $m$ and $c$,

$$
\begin{array}{ll}
K \Omega=K \Omega & G \Omega=G \Omega \\
P_{t} \Omega=0 & P_{x} \Omega=0 \\
M \Omega=m \Omega & D \Omega=c \Omega
\end{array}
$$

Notation The standard form (cf. Theorem 3.8) gives $D=\rho_{0}+(1 / m) G P_{x}+$ $1 / 2$, which shows that $\rho_{0} \Omega=(c-1 / 2) \Omega$. Hence in the following we denote $c-1 / 2$ by $\dot{c}$.

The (commuting) elements $K$ and $G$ of $\mathcal{P}$ can be used to form basis elements

$$
|j k\rangle=K^{j} G^{k} \Omega, \quad j, k \geq 0
$$

of a Fock space $\mathcal{F}=\operatorname{span}\{|j k\rangle\}$ on which $K$ and $G$ act as raising operators, while $P_{t}$ and $P_{x}$ act as lowering operators. 


\subsection{Adjoint operators and Appell systems}

The goal is to find an abelian subalgebra spanned by some selfadjoint operators acting on the representation space just constructed. Such a twodimensional subalgebra can be obtained by an appropriate "turn" of the plane $\mathcal{P}$ in the Lie algebra, namely via the adjoint action of the group element formed by exponentiating $P_{t}$. The resulting plane, $\mathcal{P}_{\beta}$ say, is abelian and is spanned by

$$
\begin{aligned}
X_{1}=e^{\beta P_{t}} K e^{-\beta P_{t}} & =\exp \left(\operatorname{ad} \beta P_{t}\right) K=K+\beta D+\beta^{2} P_{t} \\
X_{2}=e^{\beta P_{t}} G e^{-\beta P_{t}} & =G+\beta P_{x}
\end{aligned}
$$

Next we determine our canonical Appell systems. We want to compute $\exp \left(z_{1} X_{1}+z_{2} X_{2}\right) \Omega$. Setting $V_{1}=z_{1}, V_{2}=z_{2}, B_{1}=\beta$, and $B_{2}=0$ in Proposition 3.2 yields

$$
\begin{aligned}
& e^{z_{1} X_{1}} e^{z_{2} X_{2}} \Omega=e^{\beta P_{t}} e^{z_{1} K} e^{z_{2} G} e^{-\beta P_{t}} \Omega=e^{\beta P_{t}} e^{z_{1} K} e^{z_{2} G} \Omega \\
& \quad=\exp \left(\frac{z_{1} K}{1-\beta z_{1}}\right) \exp \left(\frac{z_{2} G}{1-\beta z_{1}}\right)\left(1-\beta z_{1}\right)^{-c} \exp \left(\frac{m}{2} \frac{\beta z_{2}^{2}}{1-\beta z_{1}}\right) \Omega
\end{aligned}
$$

To get the generating function for the basis $|j k\rangle$, set in equation (7)

$$
v_{1}=\frac{z_{1}}{1-\beta z_{1}}, \quad v_{2}=\frac{z_{2}}{1-\beta z_{1}}
$$

Substituting throughout, we have

Proposition 4.1 The generating function for the canonical Appell system, $\left\{|j k\rangle=K^{j} G^{k} \Omega\right\}$ is

$$
\begin{aligned}
& e^{v_{1} K} e^{v_{2} G} \Omega= \\
& \exp \left(x_{1} \frac{v_{1}}{1+\beta v_{1}}\right) \exp \left(x_{2} \frac{v_{2}}{1+\beta v_{1}}\right)\left(1+\beta v_{1}\right)^{-c} \exp \left(-\frac{m \beta}{2} \frac{v_{2}^{2}}{1+\beta v_{1}}\right)
\end{aligned}
$$

where we identify $X_{1} \Omega=x_{1} \cdot 1$ and $X_{2} \Omega=x_{2} \cdot 1$ in the realization as functions of $x_{1}, x_{2}$.

With $v_{2}=0$, we recognize the generating function for the Laguerre polynomials, while $v_{1}=0$ reduces to the generating function for Hermite polynomials. This corresponds to the results of Section 4 of [5]. 
From the exponentials $\exp \left(z_{i} X_{i}\right)$, equation (7), we identify as operators $z_{1}=$ $\partial / \partial x_{1}$ and $z_{2}=\partial / \partial x_{2}$. Using script notation for the $v_{i}$ as operators, relations (8) take the form

$$
\begin{aligned}
& \mathcal{V}_{1}=\left(1-\beta \frac{\partial}{\partial x_{1}}\right)^{-1} \frac{\partial}{\partial x_{1}} \\
& \mathcal{V}_{2}=\left(1-\beta \frac{\partial}{\partial x_{2}}\right)^{-1} \frac{\partial}{\partial x_{2}}
\end{aligned}
$$

To act on polynomials, expand $\left(1-\beta \partial / \partial x_{i}\right)^{-1}$ in geometric series

$$
\left(1-\beta \partial / \partial x_{i}\right)^{-1}=\sum_{n \geq 0} \beta^{n}\left(\frac{\partial}{\partial x_{i}}\right)^{n}
$$

So we have both a $\mathcal{V}_{1}$-Appell system and a $\mathcal{V}_{2}$-Appell system as in Section 2. The Appell space decompositions are, for $\mathcal{V}_{1}$ and $\mathcal{V}_{2}$,

$$
\begin{aligned}
& \mathcal{Z}_{n}^{(1)}=\left|\operatorname{poly}_{n}(K) \operatorname{poly}(G) \Omega\right\rangle \\
& \mathcal{Z}_{n}^{(2)}=\left|\operatorname{poly}_{n}(G) \operatorname{poly}(K) \Omega\right\rangle
\end{aligned}
$$

respectively, where poly $(\cdot)$, resp. $\operatorname{poly}_{n}(\cdot)$, denote arbitrary polynomials in the indicated variable, resp. of degree a most $n$ in the variable. Now symmetries are generated by functions of $\partial / \partial x_{1}$ and $\partial / \partial x_{2}$. We will see explicit examples in Section 6.

\subsection{Probability distributions}

Now we shall consider some probabilistic observations. We introduce an inner product such that $K^{*}=\beta^{2} P_{t}$ and $G^{*}=\beta P_{x}$. The $X_{i}$, which are formally symmetric, extend to self-adjoint operators on appropriate domains.

Expectation values are taken in the state $\Omega$, i.e., for any operator $Q$,

$$
\langle Q\rangle_{\Omega}=\langle\Omega, Q \Omega\rangle
$$

where the normalization $\langle\Omega, \Omega\rangle=1$ is understood. 
From $P_{t} \Omega=P_{x} \Omega=0$ follows that $\left\langle P_{t}\right\rangle_{\Omega}=\left\langle P_{x}\right\rangle_{\Omega}=0$ and moving $K$ and $G$ across in the inner product, that $\langle K\rangle_{\Omega}=\langle G\rangle_{\Omega}=0$ as well. Going back to equation (7), take the inner product on the left with $\Omega$. The exponential factors in $K$ and $G$ average to 1 , yielding

$$
\left\langle e^{z_{1} X_{1}} e^{z_{2} X_{2}}\right\rangle_{\Omega}=\left(1-\beta z_{1}\right)^{-c} \exp \left(\frac{m}{2} \frac{\beta z_{2}^{2}}{1-\beta z_{1}}\right)
$$

This result has an interesting probabilistic interpretation for positive values of $\beta$ and $c$. Observe that the marginal distribution of $X_{1}$ (i.e., for $z_{2}=0$ ) is gamma distribution, while the marginal distribution of $X_{2}$ (now $z_{1}=0$ ) is Gaussian. Note, however, that these are not independent random variables.

To recover the joint distribution of $X_{1}, X_{2}$, let us first recall some probability integrals (Fourier transforms):

$$
\begin{aligned}
\int_{-\infty}^{\infty} e^{i \xi y} e^{-\lambda y} y^{t-1} \lambda^{t} \theta(y) d y / \Gamma(t) & =(1-i \xi / \lambda)^{-t}, \text { for } t>0 \\
\int_{-\infty}^{\infty} e^{-i \eta u} e^{-u^{2} /(2 v)} d u & =\sqrt{2 \pi v} e^{-\eta^{2} v / 2}, \text { for } v>0
\end{aligned}
$$

where $\theta(x)$ denotes the usual Heaviside function, $\theta(x)=1$ if $x \geq 0$, zero otherwise. Replacing $z_{1}, z_{2}$ by $i z_{1}, i z_{2}$ respectively and taking inverse Fourier transforms, we have

Proposition 4.2 The joint density $p\left(x_{1}, x_{2}\right)$ of the random variables $X_{1}, X_{2}$ is given by

$$
p\left(x_{1}, x_{2}\right)=e^{-x_{1} / \beta}\left(x_{1}-\frac{x_{2}^{2}}{2 m}\right)^{\dot{c}-1} \beta^{-\dot{c}} \theta\left(x_{1}-\frac{x_{2}^{2}}{2 m}\right) \frac{d x_{1} d x_{2}}{\Gamma(\dot{c}) \sqrt{2 \pi m \beta}}
$$

for $c, \beta>0$, where $\dot{c}=c-1 / 2$.

In the first factor, writing $x_{1}=x_{1}-x_{2}^{2} /(2 m)+x_{2}^{2} /(2 m)$ shows where the Gaussian factor comes in. The result says that the marginal distribution of $X_{2}$ is Gaussian with mean 0 and variance $2 m \beta$. Conditional on $X_{2}, X_{1}$ is gamma with parameters $1 / \beta$ and $c-1 / 2$ taking values in the interval $\left(x_{2}^{2} /(2 m), \infty\right)$. In the special case $c=1 / 2$, i.e., $\dot{c}=0$, the gamma density collapses to a delta function: $\delta\left(x_{1}-x_{2}^{2} /(2 m)\right)$. 


\section{$5 \quad$ Leibniz function and orthogonal basis}

Once the Leibniz formula for our Lie algebra $\mathcal{S}_{1}$ is known (Proposition 3.2), we can proceed to define coherent states, find the Leibniz function - inner product of coherent states - and show that we have a Hilbert space with self-adjoint commuting operators $X_{1}=P_{t}+D+K$ and $X_{2}=G+P_{x}$ (here the $\beta$ in equations (6) is set equal to 1 ). We recover the raising and lowering operators as elements of the Lie algebra acting on the Hilbert space with basis consisting of the canonical Appell system.

The two-parameter family of coherent states is defined as

$$
\psi_{V}=\psi_{V_{1}, V_{2}}=e^{V_{1} K} e^{V_{2} G} \Omega
$$

Using Proposition 3.2, we see

Proposition 5.1 With $K^{*}=P_{t}$ and $G^{*}=P_{x}$, the Leibniz function is

$$
\Upsilon_{B V}=\left(1-B_{1} V_{1}\right)^{-c} \exp \left(\frac{m}{2} \frac{B_{1} V_{2}^{2}+2 B_{2} V_{2}+B_{2}^{2} V_{1}}{1-B_{1} V_{1}}\right)
$$

Proof: Use Proposition 3.2 in the relation

$$
\Upsilon_{B V}=\left\langle\psi_{B}, \psi_{V}\right\rangle=\left\langle\Omega, e^{B_{2} P_{x}} e^{B_{1} P_{t}} e^{V_{1} K} e^{V_{2} G} \Omega\right\rangle
$$

Note that the Leibniz function is symmetric in $B$ and $V$, which is equivalent to the inner product being symmetric, and thus the Hilbert space being well-defined.

It is remarkable that the Lie algebra can be reconstructed from the Leibniz function $\Upsilon_{B V}$. The idea is that differentiation $\Upsilon_{B V}$ with respect to $V_{1}$ brings down $K$ acting on $\psi_{V}$, while differentiation with respect to $B_{1}$ brings down a $K$ acting on $\psi_{B}$ which moves across the inner product as $P_{t}$ acting on $\psi_{V}$. Similarly for $G$ and $P_{x}$. We thus introduce canonical bosons, creation operators $\mathcal{R}_{i}$, and annihilation (velocity) operators $\mathcal{V}_{i}$, satisfying $\left[\mathcal{V}_{i}, \mathcal{R}_{j}\right]=\delta_{i j}$. We thus identify $K=\mathcal{R}_{1}, G=\mathcal{R}_{2}$. Note, however, that $\mathcal{V}_{1}$ is not the adjoint of $\mathcal{R}_{1}$, nor $\mathcal{V}_{2}$ that of $\mathcal{R}_{2}$. In fact, our goal is to determine the boson realization 
of $P_{t}$ and $P_{x}$, the respective adjoints.

Here is a method to find the boson realization. First, one determines the partial differential equations for $\Upsilon=\Upsilon_{B V}$ :

$$
\begin{aligned}
& \frac{\partial \Upsilon}{\partial B_{1}}=V_{1}^{2} \frac{\partial \Upsilon}{\partial V_{1}}+V_{1} V_{2} \frac{\partial \Upsilon}{\partial V_{2}}+c V_{1} \Upsilon+\frac{m}{2} V_{2}^{2} \Upsilon \\
& \frac{\partial \Upsilon}{\partial B_{2}}=V_{1} \frac{\partial \Upsilon}{\partial V_{2}}+m V_{2} \Upsilon
\end{aligned}
$$

Then, one interprets each multiplication by $V_{i}$ as the operator $\mathcal{V}_{i}$ and each differentiation by $V_{i}$ as the operator $\mathcal{R}_{i}$. This gives the following action of the operators $P_{x}$ and $P_{t}$ on polynomial functions of $K$ and $G$ :

$$
P_{x}=m \mathcal{V}_{2}+\mathcal{R}_{2} \mathcal{V}_{1}, \quad P_{t}=c \mathcal{V}_{1}+\mathcal{R}_{1} \mathcal{V}_{1}^{2}+\frac{m}{2} \mathcal{V}_{2}^{2}+\mathcal{R}_{2} \mathcal{V}_{1} \mathcal{V}_{2}
$$

This means that $P_{x}$ acts on $|j k\rangle=\mathcal{R}_{1}^{j} \mathcal{R}_{2}^{k}|00\rangle$ as follows

$$
P_{x}|j k\rangle=m k|j, k-1\rangle+j|j-1, k+1\rangle
$$

and $P_{t}$ does similarly. The element $D$ is recovered via

$$
D=\left[P_{t}, K\right]=\left[c \mathcal{V}_{1}+\mathcal{R}_{1} \mathcal{V}_{1}^{2}+\frac{m}{2} \mathcal{V}_{2}^{2}+\mathcal{R}_{2} \mathcal{V}_{1} \mathcal{V}_{2}, \mathcal{R}_{1}\right]=c+2 \mathcal{R}_{1} \mathcal{V}_{1}+\mathcal{R}_{2} \mathcal{V}_{2}
$$

Summarizing, we have

Theorem 5.2 The representation of the Schrödinger algebra on the Fock space $\mathcal{F}$ with basis $|j k\rangle=K^{j} G^{k} \Omega$ is given by

$$
\begin{aligned}
K & =\mathcal{R}_{1} \\
G & =\mathcal{R}_{2} \\
P_{x} & =m \mathcal{V}_{2}+\mathcal{R}_{2} \mathcal{V}_{1} \\
P_{t} & =c \mathcal{V}_{1}+\mathcal{R}_{1} \mathcal{V}_{1}^{2}+\frac{m}{2} \mathcal{V}_{2}^{2}+\mathcal{R}_{2} \mathcal{V}_{1} \mathcal{V}_{2} \\
D & =c+2 \mathcal{R}_{1} \mathcal{V}_{1}+\mathcal{R}_{2} \mathcal{V}_{2} \\
M & =m
\end{aligned}
$$

Corollary 5.3 In the above representation, the Schrödinger operator $\mathbf{S}=$ $P_{t}-P_{x}^{2} /(2 m)$ is represented by

$$
\mathbf{S}=\dot{c} \mathcal{V}_{1}+\mathcal{R}_{o} \mathcal{V}_{1}^{2}
$$

where we define $\mathcal{R}_{0}=\mathcal{R}_{1}-\mathcal{R}_{2}^{2} /(2 m)$, cf. Theorem 3.8. 
A very important feature of the Leibniz function $\Upsilon_{B V}$ is that it is the generating function for the inner products of the elements of the basis. Indeed, expanding the exponentials defining the coherent states yields

$$
\Upsilon_{B V}=\sum_{j, k, j^{\prime}, k^{\prime}}\left\langle j k \mid j^{\prime} k^{\prime}\right\rangle \frac{B_{1}^{j} B_{2}^{k} V_{1}^{j^{\prime}} V_{2}^{k^{\prime}}}{j ! k ! j^{\prime} ! k^{\prime} !}
$$

For an orthogonal basis, a necessary and sufficient condition is that this must be a function only of the pair products $B_{1} V_{1}$ and $B_{2} V_{2}$. We proceed to find an orthogonal basis.

Lemma 5.4 The Leibniz function can be expressed as

$$
\Upsilon_{B V}=\left(1-B_{1} V_{1}\right)^{-\dot{c}} \exp \left(\frac{B_{1}}{2 m} \frac{\partial^{2}}{\partial B_{2}^{2}}+\frac{V_{1}}{2 m} \frac{\partial^{2}}{\partial V_{2}^{2}}\right) e^{m B_{2} V_{2}}
$$

with $\dot{c}=c-1 / 2$.

Proof: In the formulation of Corollary 3.10 first set $B_{2}=0$. Then use the special realization as in equation (5) with $x=B_{2}$. As in Corollary 3.10

$$
\begin{array}{r}
\exp \left(\frac{B_{1}}{2 m} \frac{d^{2}}{d B_{2}^{2}}\right) \exp \left(V_{1} \frac{m B_{2}^{2}}{2}+V_{2} m B_{2}\right) \\
=\exp \left(\frac{V_{1}}{1-B_{1} V_{1}} \frac{m B_{2}^{2}}{2}+\frac{V_{2}}{1-B_{1} V_{1}} m B_{2}\right) \\
\quad \times\left(1-B_{1} V_{1}\right)^{-1 / 2} \exp \left(\frac{m}{2} \frac{B_{1} V_{2}^{2}}{1-B_{1} V_{1}}\right)
\end{array}
$$

which combines to yield $\Upsilon_{B V}$ up to the factor $\left(1-B_{1} V_{1}\right)^{-\dot{c}}$. Now observe that

$$
e^{m B_{2} V_{2}+(m / 2) V_{1} B_{2}^{2}}=\exp \left(\frac{V_{1}}{2 m} \frac{\partial^{2}}{\partial V_{2}^{2}}\right) e^{m B_{2} V_{2}}
$$

where on $\exp \left(m B_{2} V_{2}\right), \partial / \partial V_{2}$ acts simply as multiplication by $m B_{2}$. Combining with the above observations yields the result.

Now for the main result (expressed in terms of $R_{0}=K-G^{2} /(2 m)$, see Corollary 5.3) 
Theorem 5.5 The set $|a b\rangle=R_{0}^{a} G^{b} \Omega, a, b \geq 0$, forms an orthogonal basis with squared norms equal

$$
\langle a b \mid a b\rangle=(\dot{c})_{a} a ! b ! m^{b}
$$

where $(\dot{c})_{a}=\dot{c}(\dot{c}+1) \cdots(\dot{c}+a-1)$.

Proof: From Lemma 5.4,

$$
\begin{aligned}
(1 & \left.-B_{1} V_{1}\right)^{-\dot{c}} e^{m B_{2} V_{2}} \\
& =\exp \left(-\frac{B_{1}}{2 m} \frac{\partial^{2}}{\partial B_{2}^{2}}-\frac{V_{1}}{2 m} \frac{\partial^{2}}{\partial V_{2}^{2}}\right)\left\langle e^{B_{1} K+B_{2} G} \Omega, e^{V_{1} K+V_{2} G} \Omega\right\rangle \\
& =\left\langle e^{\left.B_{1}\left(K-G^{2} /(2 m)\right)+B_{2} G\right)} \Omega, e^{V_{1}\left(K-G^{2} /(2 m)\right)+V_{2} G} \Omega\right\rangle \\
& =\left\langle e^{B_{1} R_{0}+B_{2} G} \Omega, e^{V_{1} R_{0}+V_{2} G} \Omega\right\rangle
\end{aligned}
$$

Now we have the generating function for the inner products $\left\langle a b \mid a^{\prime} b^{\prime}\right\rangle$ depending only on the pair products $B_{1} V_{1}, B_{2} V_{2}$. Hence orthogonality. Expanding the left-hand side of the equation yields the squared norms.

Similarly, we have for the canonical Appell system,

Proposition 5.6 Let $X_{0}=X_{1}-X_{2}^{2} /(2 m)$, with the identification $X_{0} \Omega=x_{0} \cdot 1$. Then

$$
e^{v_{0} R_{0}} e^{v_{2} G} \Omega=\exp \left(x_{0} \frac{v_{0}}{1+\beta v_{0}}\right)\left(1+\beta v_{0}\right)^{-\dot{c}} \exp \left(x_{2} v_{2}-\beta m v_{2}^{2} / 2\right)
$$

Proof: $\quad$ First substitute $v_{0}$ for $v_{1}$ in Proposition 4.1. And observe that

$$
e^{v_{0} R_{0}} e^{v_{2} G} \Omega=\exp \left(-\frac{v_{0}}{2 m} \frac{\partial^{2}}{\partial v_{2}^{2}}\right) e^{v_{0} K} e^{v_{2} G} \Omega
$$

Now use the special realization, equation (5), taking $x=v_{2}$ in Corollary 3.10, with

$$
B_{1}=-v_{0}, \quad B_{2}=0, \quad V_{1}=-\frac{\beta}{1+\beta v_{0}}, \quad V_{2}=\frac{x_{2} / m}{1+\beta v_{0}}
$$

After substituting accordingly and simplifying, one finds the stated result.

Note that now the system decouples into Laguerre polynomials in the variable $x_{0}$ and Hermite polynomials in the variable $x_{2}$. 


\section{Evolution equations and Appell systems}

We shall now consider evolutions on the 2-dimensional space $M$ spanned by $G$ and $R_{0}$ in terms of scalar functions $u$ on $M$ parametrized by time $\tau$. Note that polynomial functions on $M$ can be interpreted as vectors of the Fock space $\mathcal{F}=\operatorname{span}\left\{|j k\rangle=K^{j} G^{k} \Omega\right\}$ of the representation of the Schrödinger algebra. The evolution equation is

$$
u_{\tau}=H u
$$

where the subscript $\tau$ denotes differentiation over the time parameter. For generators of evolutions, we shall consider operator functions $H=H\left(P_{t}, P_{x}\right)$. In order to have an operator calculus, the function $H$ is chosen so that $H\left(\partial / \partial x_{1}, \partial / \partial x_{2}\right)$ generates a Lévy process on $\mathbf{R}^{2}$, with its associated convolution semigroup of probability measures providing the fundamental solution to the evolution equation (9). Taking such a process $w(\tau)=\left(w_{1}(\tau), w_{2}(\tau)\right)$ with generator $H$, consider the action of the group element $\exp \left(w_{1}(\tau) P_{x}+w_{2}(\tau) P_{t}\right)$ as an operator on functions of $K$ and $G$. Then the (partial) group law yields the solution to the evolution equation $u_{\tau}=H u$ with elements of the basis $|j k\rangle=K^{j} G^{k} \Omega$ as initial functions. These are Appell systems on the Schrödinger algebra. In this case, since the elements $P_{x}$ and $P_{t}$ commute, the process considered on the group is essentially the same as that in Euclidean space, and we have an operator calculus for the operators $P_{t}$ and $P_{x}$ via the generator of the process $H$.

As we have seen in the previous section, it is convenient to use $R_{0}$ instead

of $K$, so we will take as initial functions $|a b\rangle=R_{0}^{a} G^{b} \Omega$. A special case of Proposition 3.2, with $B_{2}=V_{2}=0$ gives the Leibniz formula for sl(2), namely

$$
e^{w_{1} L_{0}} e^{v_{0} R_{0}}=\exp \left(\frac{v_{0}}{1-v_{0} w_{1}} R_{0}\right)\left(1-v_{0} w_{1}\right)^{-\rho_{0}} \exp \left(\frac{w_{1}}{1-v_{0} w_{1}} L_{0}\right)
$$

Similarly, taking in the same formula $B_{1}=V_{1}=0$, yields the Leibniz formula for the $\mathrm{HW}$-algebra, which we want in the form

$$
\exp \left(w_{2} P_{x}\right) \exp \left(v_{2} G\right)=\exp \left(m w_{2} v_{2}\right) \exp \left(v_{2} G\right) \exp \left(w_{2} P_{x}\right)
$$

Thus, 
Proposition 6.1 The abelian subgroup generated by $P_{t}$ and $P_{x}$ on the basis $|a b\rangle$ has generating function

$$
\begin{aligned}
& e^{w_{1} P_{t}+w_{2} P_{x}} e^{v_{0} R_{0}+v_{2} G} \Omega= \\
& \quad \exp \left(\frac{v_{0}}{1-v_{0} w_{1}} R_{0}\right)\left(1-v_{0} w_{1}\right)^{-\dot{c}} \exp \left(v_{2}\left(G+m w_{2}\right)+m w_{1} v_{2}^{2} / 2\right)
\end{aligned}
$$

Proof: $\quad$ First, use the fact that $P_{x}$ commutes with $R_{0}$ together with the Leibniz formula for the HW-algebra to yield

$$
e^{w_{1} P_{t}} e^{v_{0} R_{0}} e^{v_{2} G} e^{m w_{2} v_{2}} \Omega
$$

Now write, using the standard form, Theorem 3.8, $L=L_{0}+L_{1}$, with $L_{1}=$ $P_{x}^{2} /(2 m)$. Since $L_{1}$ commutes with $R_{0}$, we have

$$
e^{w_{1} L_{0}} e^{v_{0} R_{0}} e^{w_{1} L_{1}} e^{v_{2} G} e^{m w_{2} v_{2}} \Omega
$$

We can reconstitute $L_{1}$ as $P_{t}$ since $L_{0}$ commutes with $G$, and use Proposition 3.2 , or, equivalently, think of $P_{x}$ as acting formally like $m \frac{d}{d G}$. In any case, we arrive at

$$
e^{w_{1} L_{0}} e^{v_{0} R_{0}} e^{w_{1} L_{1}} e^{v_{2} G} e^{m w_{2} v_{2}+m w_{1} v_{2}^{2} / 2} \Omega
$$

Now use the Leibniz formula for $\operatorname{sl}(2)$ mentioned above, and since the $\rho_{0}$ and $L_{0}$ commute with $G$, and $\rho_{0} \Omega=\dot{c} \Omega$, the stated result follows.

Finally, the solution to the evolution equation $u_{\tau}=H u$ is found by averaging over $w_{1}$ and $w_{2}$, i.e., the angle brackets on the right-hand side denoting expected value,

$e^{\tau H\left(P_{t}, P_{x}\right)}|a b\rangle=\left\langle\exp \left(\frac{v_{0}}{1-v_{0} w_{1}} R_{0}\right)\left(1-v_{0} w_{1}\right)^{-\dot{c}} \exp \left(v_{2}\left(G+m w_{2}\right)+m w_{1} v_{2}^{2} / 2\right)\right\rangle$

Expanding in $v_{0}, v_{2}$ and evaluating moments of the process yields the corresponding Appell systems.

For $w_{1}, w_{2}$ independent Gaussians with mean zero and variance $\tau$, we have $H\left(P_{t}, P_{x}\right)=\frac{1}{2}\left(P_{t}^{2}+P_{x}^{2}\right)$, with $u_{\tau}=H u$ a natural extension of the classical heat equation. 


\section{References}

[1] Ballesteros A Herranz F J and Parashar P: (1+1) Schrödinger Lie bialgebras and their Poisson-Lie groups, J. Phys. A: Math. Gen. 33, 2000, pp. 3445-3465.

[2] Barut A O and Rączka R: Theory of group representations and applications, 2nd ed., PWN, Warszawa, 1980.

[3] Barut A O and $\mathrm{Xu}$ B-W: Conformal covariance and the probability interpretation of wave equations, Phys. Lett., 82A, 5, 1981, pp. 218 220 .

[4] Burdet G, Patera J, Perrin M and Winternitz P: The optical group and its subgroups, J. Math. Phys., 19, 1978, pp. 1758-1780.

[5] Dobrev V K, Doebner H D, and Mrugalla Ch: Lowest weight representations of the Schrödinger algebra and generalized heat/Schrödinger equations, Rep. Math. Phys., 39, 2, 1997, pp. 201-218.

[6] Feinsilver P and Schott R: Appell systems on Lie groups, J. Th. Prob., 5, 2, 1992, pp. 251-281.

[7] Feinsilver P and Schott R: Lie response to signals with noise, Probability on algebraic structures, Contemporary Math., 261, AMS, 2000, pp. 1522 .

[8] Hagen C R: Scale and conformal transformations in Galilean-covariant field theory, Phys. Rev. D5, 2, 1972, pp. 377-388.

[9] Hecht K T: The vector coherent state method and its application to problems of higher symmetries, Springer Lect. Notes in Physics, 290, 1987.

[10] Niederer U: The maximal kinematical invariance group of the free Schrödinger equation, Helv. Phys. Acta, 45, 1972/73, pp. 802-810. 